\title{
The static and dynamic screening of power loss of a two-dimensional electron gas
}

\author{
C. Bennett, N. Balkan† \\ University of Essex, Department of Physics, Colchester, U.K. \\ B. TANATAR \\ Bilkent University, Department of Physics, 06532 Ankara, Turkey \\ H. Celik, M. Cankurtaran \\ Hacettepe University, Department of Physics, 06533 Ankara, Turkey
}

(Received 24 September 1997)

Experimental results concerning the well-width dependence of the acoustic-phonon-assisted energy relaxation of a two-dimensional electron gas in $\mathrm{GaAs} / \mathrm{Ga}_{1-x} \mathrm{Al}_{x}$ As quantum-well structures are compared with theoretical models that involve piezoelectric and deformationpotential scattering and the effects of static and dynamic screening of the electron-acoustic phonon interaction. It is shown that screening only slightly modifies the predictions of the approximate calculations.

(c) 1998 Academic Press

Key words: 2D warm electrons, energy relaxation, screening, power loss.

\section{Introduction}

Experimental work concerning the energy loss rate of the two-dimensional (2D) electrons in the acoustic phonon scattering regime has been reported by a large number of groups [1-7]. It has often been pointed out that experimental results do not agree well with the theoretical predictions. Recently, we have also reported power-loss results in $\mathrm{GaAs} / \mathrm{Ga}_{1-x} \mathrm{Al}_{x}$ As quantum-well structures obtained as a function of well width, and addressed a number of uncertainties associated with the cooling mechanisms [8]. It was argued that one reason for the discrepancy between the theory and experiment might be associated with the over-estimated piezoelectric component in 2D. The aim of this paper is, therefore, to understand whether the inclusion of the screening of electron-acoustic phonon interaction by the degenerate $2 \mathrm{D}$ electron gas may account for the disagreement between experiment and theory.

\section{Experimental results}

Most of the experimental results and the details of the theoretical model have been reported by us [8]. We, therefore, give only a brief summary for convenience.

$\dagger$ Author to whom correspondence should be addressed. 


\begin{tabular}{|c|c|c|c|c|c|c|c|c|}
\hline Sample & $\begin{array}{l}\text { No. of } \\
\text { wells }\end{array}$ & $\begin{array}{l}\text { Well } \\
\text { width } \\
(\AA)\end{array}$ & $\begin{array}{c}\mathrm{Al} \\
\text { concentration } \\
(\%)\end{array}$ & $\begin{array}{l}\text { 2D electron } \\
\text { density } \\
\left(\mathrm{cm}^{-2}\right)\end{array}$ & $\begin{array}{c}\text { First } \\
\text { subband } \\
E_{1} \\
(\mathrm{meV})\end{array}$ & $\begin{array}{c}\text { Fermi } \\
\text { energy: } \\
E_{F}-E_{1} \\
(\mathrm{meV})\end{array}$ & $\begin{array}{l}\text { Hall mobility } \\
\mu(4.2 \mathrm{~K}) \\
\left(\mathrm{cm}^{2}(\mathrm{Vs})^{-1}\right)\end{array}$ & $\begin{array}{c}\text { Base } \\
\text { lattice } \\
\text { temperature } \\
T_{0}(\mathrm{~K})\end{array}$ \\
\hline A & 10 & 51 & 32 & $9.0 \times 10^{12}$ & 73.7 & 32.2 & $2.43 \times 10^{4}$ & 1.55 \\
\hline B & 10 & 75 & 33 & $1.1 \times 10^{13}$ & 45.5 & 39.3 & $2.98 \times 10^{4}$ & 1.70 \\
\hline $\mathrm{C}$ & 10 & 78 & 32 & $9.9 \times 10^{12}$ & 43.1 & 35.4 & $3.75 \times 10^{4}$ & 1.64 \\
\hline $\mathrm{D}$ & 10 & 106 & 32 & $9.1 \times 10^{12}$ & 27.7 & 32.5 & $2.42 \times 10^{4}$ & 1.70 \\
\hline $\mathrm{E}$ & 10 & 145 & 32 & $1.08 \times 10^{13}$ & 16.9 & 38.6 & $2.58 \times 10^{4}$ & 1.57 \\
\hline
\end{tabular}

The parameters of the $\mathrm{GaAs} / \mathrm{Ga}_{1-x} \mathrm{Al}_{x} \mathrm{As}$ multiple-quantum-well samples used in the investigations are given in Table 1. All the samples were grown with the molecular beam epitaxy (MBE) technique. Samples were fabricated in the form of Hall-bar shapes and the ohmic contacts were formed by alloying Au/Ge/Ni. The experiments involved the measurements of the Shubnikov de Haas $(\mathrm{SdH})$ oscillations as a function of both the applied electric field at a fixed lattice temperature (the lowest of the lattice temperature range, i.e. $T_{0}$ in Table 1, and the lattice temperature $\left(T_{0}<T_{L}<4.2 \mathrm{~K}\right)$ at a fixed electric field that was low enough to ensure ohmic conditions and hence to avoid carrier heating. Thermal damping of the oscillations, at a fixed magnetic field was determined only by the temperature, magnetic field and the effective electron mass as described in [8-11], i.e.

$$
\frac{A\left(T_{L}\right)}{A\left(T_{0}\right)}=\frac{T_{L} \sinh \left(2 \pi^{2} k_{B} T_{0} m^{*} / \hbar e B\right)}{T_{0} \sinh \left(2 \pi^{2} k_{B} T_{L} m^{*} / \hbar e B\right)}
$$

where $A\left(T_{L}\right)$ and $A\left(T_{0}\right)$ are the amplitudes of the oscillations at the two lattice temperatures $T_{L}$ and $T_{0}$ respectively. The change in the amplitude of the $\mathrm{SdH}$ oscillations with electric field can be described in terms of the electric-field-induced electron heating [11], then in eqn (1), $T_{L}$ can be replaced by the electron temperature, $T_{e}$, and therefore, electron temperatures can be determined by directly comparing the relative amplitudes of the oscillations plotted as a function of the electric field and lattice temperature [10, 11]:

$$
\left[\frac{A\left(T_{L}\right)}{A\left(T_{0}\right)}\right]_{F=F_{0}}=\left[\frac{A(E)}{A\left(E_{0}\right)}\right]_{T_{l}=T_{0}}
$$

where $E_{0}$ and $T_{0}$ are the lowest applied electric field and the lattice temperature of the measurements. The power loss from the electrons to the lattice by the emission of acoustic phonons can be calculated from the steady-state energy balance equation via [11],

$$
p=e \mu(E)^{2}
$$

where $p, \mu$ and $E$ are the energy loss/supply rate per electron, electric-field-dependent electron mobility, and the applied electric field respectively.

The power loss versus electron temperature is plotted in Fig. 1 for all the samples studied. It is evident from the figure that both the magnitude and the electron temperature dependence of the power loss vary significantly from one sample to another. Since both the barrier parameters and the 2D electron densities of all the samples are identical within $10 \%$, the observed variation is indicative of an association with the only variable parameter in the samples; i.e. the quantum-well width, $L_{Z}$. In order to show the well-width dependence clearly, in Fig. 2 we plotted the power loss as a function of the well width at $T_{e}=4 \mathrm{~K}$. The power loss changes by about a factor of two in the range of quantum wells studied. There is a clear reduction in the power loss with increasing well width up to $L_{Z}=106 \AA$. However, although very limited data are available 


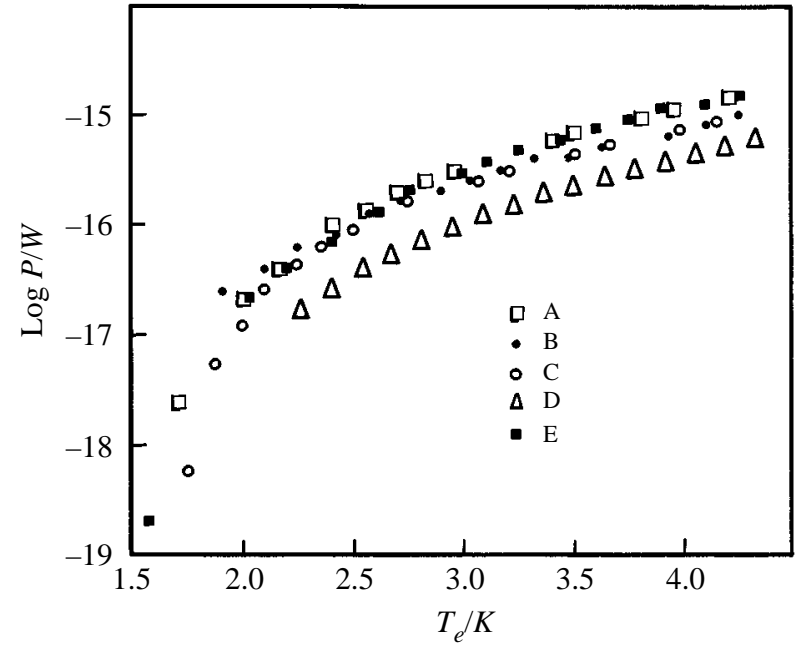

Fig. 1. Energy loss rate per electron versus electron temperatures for the samples studied. The letters refer to samples in Table 1.

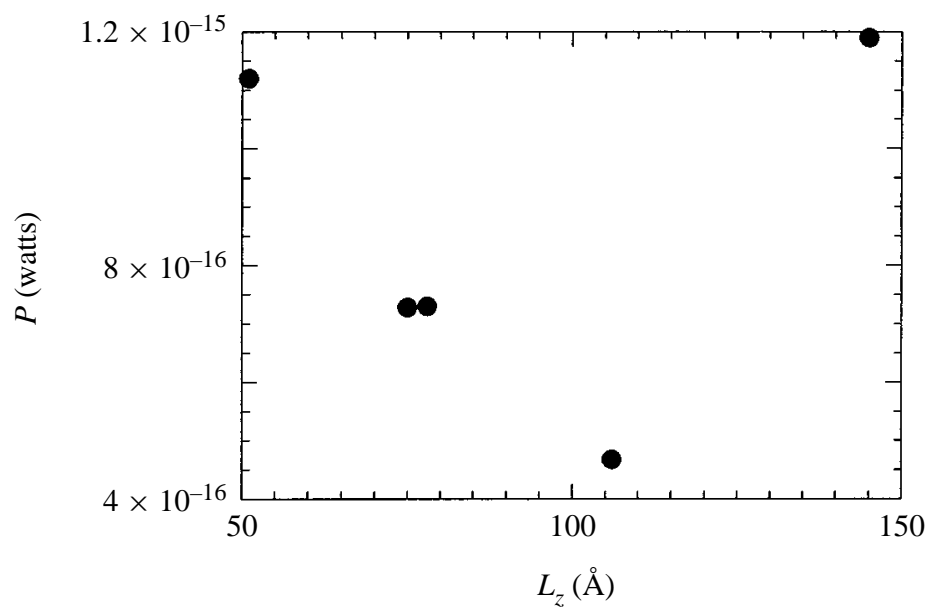

Fig. 2. Quantum-well width dependence of the electron power loss at electron temperature $T_{e}=4 \mathrm{~K}$.

for wider wells $\left(L_{z} \geq 100 \AA\right.$ ) the indication is that the power loss tends to increase as the confinement length increases and as the electrons acquire more of a quasi-three-dimensional (3D) characteristic.

\section{Theoretical models and discussions}

\subsection{Approximate results for the unscreened interaction}

Approximate analytical expressions for the unscreened interactions in $3 \mathrm{D}$ and $2 \mathrm{D}$ are given in $[8,12,13]$. The power loss due to scattering by acoustic phonons is then calculated using these expressions in two distinct temperature regimes. 


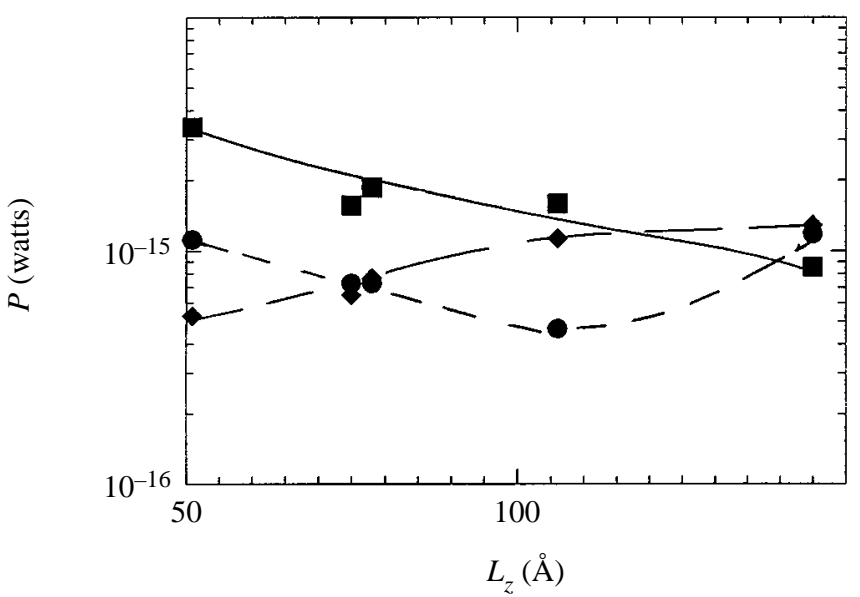

Fig. 3. Well-width dependence of the theoretical and the experimental power loss at $T_{e}=4 \mathrm{~K}$. Full circles: experimental results; full squares: approximate solution of the 2D analytical model; full diamond: approximate solution of the 3D analytical model. Lines are to guide the eye.

1. The equipartition regime, where

$$
\frac{\hbar \omega_{q}}{k T} \ll 1
$$

and, hence the phonon distribution $n\left(\omega_{q}\right)$ is:

$$
n\left(\omega_{q}\right)=\frac{1}{\exp \left(\hbar \omega_{q} / k_{B} T\right)-1} \approx \frac{k_{B} T}{\hbar \omega_{q}}
$$

where $\hbar \omega_{q}$ is the acoustic phonon energy at wavevector $q$.

2. A low-temperature (Bloch-Gruneisen) regime where the phonon population diminishes and the Pauli exclusion increasingly restricts the allowed process:

$$
n\left(\omega_{q}\right) \ll 1 .
$$

The scattering by absorption is negligible and only spontaneous emission is important.

In Fig. 3 we have plotted the total (polar + non-polar) theoretical power loss per electron for all the samples at $T_{e}=4 \mathrm{~K}$ and at base lattice temperatures $T_{0}$ as listed in Table 1 . Other parameters used in the calculations are: $T_{e}=4 \mathrm{~K} ; n=1.0 \times 10^{16} \mathrm{~m}^{-2} ; \rho=5360 \mathrm{~kg} \mathrm{~m}^{-3} ; \Xi=7 \mathrm{eV} ; \kappa_{a v}^{2}=2.52 \times 10^{-3} ; v_{s}=5240 \mathrm{~ms}^{-1}$; $\varepsilon=13.18 ; m^{*}=0.07 \mathrm{~m}_{e}$. Although the use of the bulk approximation for the smaller quantum wells is questionable, for the widest well, where the electron gas has less of a $2 \mathrm{D}$ character the power loss is described better with the 3D model. On the other hand, despite failing to predict the magnitude of the experimental power loss, the 2D model has a well-width dependence very similar to the experimental results for the narrow wells. Neither the 2D nor the 3D model explain the observations for the whole range of measurements.

\subsection{Exact calculations and the static and dynamic screening of the interaction}

Since the well-width dependence of the experimental results is not explained by the approximate results of usual scattering theory, we now include the effect of the electron gas in screening the electron-acoustic phonon 
interaction. We start with the general scattering equation from many-body theory using the approximation of one subband [14],

$$
P=\frac{2}{\hbar} \sum_{\boldsymbol{q}_{\|}} \int_{0}^{\infty} d(\hbar \omega) \hbar \omega\left[n_{T_{e}}(\omega)-n_{T}(\omega)\right] \operatorname{Im}\left[V_{s c r}(\boldsymbol{q}, \omega)\right] \operatorname{Im}\left[\chi\left(\boldsymbol{q}_{\|}, \omega\right)\right],
$$

where $T$ is the lattice temperature, $T_{e}$ is the electron temperature, $\chi\left(\boldsymbol{q}_{\|}, \omega\right)$ is the reducible polarizability function given by $\chi_{0}\left(\boldsymbol{q}_{\|}, \omega\right) / \varepsilon\left(\boldsymbol{q}_{\|}, \omega\right), \varepsilon\left(\boldsymbol{q}_{\|}, \omega\right)=1-V\left(\boldsymbol{q}_{\|}\right) \chi_{0}\left(\boldsymbol{q}_{\|}, \omega\right)$ is the dielectric function and

$$
V_{S C R}\left(\boldsymbol{q}_{\|}, \omega\right)=\frac{V_{p h}\left(\boldsymbol{q}_{\|}, \omega\right)}{1-\left[V\left(\boldsymbol{q}_{\|}\right)+V_{p h}\left(\boldsymbol{q}_{\|}, \omega\right)\right] \chi\left(\boldsymbol{q}_{\|}, \omega\right)} .
$$

Here $V\left(\boldsymbol{q}_{\|}\right)$is the 2D Fourier transform of the Coulomb matrix element and $V_{p h}\left(\boldsymbol{q}_{\|}, \omega\right)=$ $\sum_{q_{z}}|M(\boldsymbol{q})|^{2} D\left(\boldsymbol{q}_{\|}, \omega\right)$ is the phonon potential. The matrix element is given by

$$
|M(\boldsymbol{q})|^{2}=\frac{\hbar}{2 \rho V_{c} \omega_{\boldsymbol{q}}}\left[\Xi^{2} q^{2}+\frac{K_{a v}^{2} c_{L} e^{2}}{\varepsilon_{0} \varepsilon_{s}}\right]\left[\frac{\sin \left(q_{z} L_{z} / 2\right)}{q_{z} L_{z} / 2}+\frac{\sin \left(q_{z} L_{z} / 2+\pi\right)}{q_{z} L_{z}+2 \pi}+\frac{\sin \left(q_{z} L_{z} / 2-\pi\right)}{q_{z} L_{z}-2 \pi}\right]^{2},
$$

where the first part is for the interaction with acoustic phonons by deformation potential and the second part by piezoelectric scattering, and $D(\boldsymbol{q}, \omega)$ is the acoustic phonon propagator,

$$
D(\boldsymbol{q}, \omega)=\frac{2 \omega_{\boldsymbol{q}}}{\hbar\left(\omega^{2}-\omega_{\boldsymbol{q}}^{2}\right)} .
$$

Also, the acoustic modes have the dispersion $\omega_{q}=v_{s} q$ and

$$
\chi_{0}\left(\boldsymbol{q}_{\|}, \omega\right)=2 \sum_{\boldsymbol{k}_{\|}} \frac{f\left(E_{\boldsymbol{k}_{\|}}\right)-f\left(E_{\boldsymbol{k}_{\|} \boldsymbol{q}_{\|}}\right)}{\hbar(\omega+i \gamma)-E_{\boldsymbol{k}_{\|}+\boldsymbol{q}_{\|}}+E_{\boldsymbol{k}_{\|}}} .
$$

We can simplify (6) by assuming that the acoustic phonons do not couple because they have much less energy than the plasmons, i.e. eqn (7) becomes $V_{s c r}\left(\boldsymbol{q}_{\|}, \omega\right)=V_{p h}\left(\boldsymbol{q}_{\|}, \omega\right)$. The imaginary part of the phonon propagator becomes a delta function, setting $\omega$ to $\omega_{q}$, leaving [15]

$$
P=\sum_{\boldsymbol{q}} 2 \omega_{\boldsymbol{q}}\left[n_{T_{e}}\left(\omega_{\boldsymbol{q}}\right)-n_{T}\left(\omega_{\boldsymbol{q}}\right)\right]|M(\boldsymbol{q})|^{2} \operatorname{Im}\left[-\chi\left(\boldsymbol{q}_{\|}, \omega_{\boldsymbol{q}}\right)\right] .
$$

If we replace $\varepsilon\left(\boldsymbol{q}_{\|}, \omega_{\boldsymbol{q}}\right)$ with $\varepsilon\left(\boldsymbol{q}_{\|}, 0\right)$ the static screening result is obtained. For acoustic phonons this should also be a good approximation, again because $\omega_{q} \ll \omega_{P}$, where $\omega_{p}$ is the plasmon energy. We will compare both the dynamic and static results. If $\varepsilon\left(\boldsymbol{q}_{\|}, \omega_{q}\right)$ is set to unity then (11) gives the exact unscreened power loss.

In Fig. 4 the results of the unscreened exact, together with the screened theoretical, power-loss calculations are compared with the experimental results. It can be seen that the exact result has a much shallower gradient than the approximate result used above and in earlier treatments [8], which actually has a better comparison with the experimental results. This is due to the approximation of ignoring the matrix element in the perpendicular direction and setting the associated perpendicular wavevector, $q_{z}$, to zero. In the exact result the wavevector does have a dependence on well width and hence the approximation, which follows $1 / L_{Z}$, does not produce the correct well-width dependence. The effect of screening has only reduced the rates; the gradient is about the same. Both static and dynamic screening give similar results, as expected above, but both are now lower than the experimental data and neither have the same form. The reduction in the power loss may be explained by how the value for the deformation potential is obtained. In all the theoretical calculations the value of $7 \mathrm{eV}$ was used for $\Xi$. This value was obtained by fitting the theory with the experimental data for the bulk power loss using similar methods [16]. However, no screening was included when this fitting was done and hence the value for the deformation potential is too low for the case of screening. Other authors [17] have used larger 


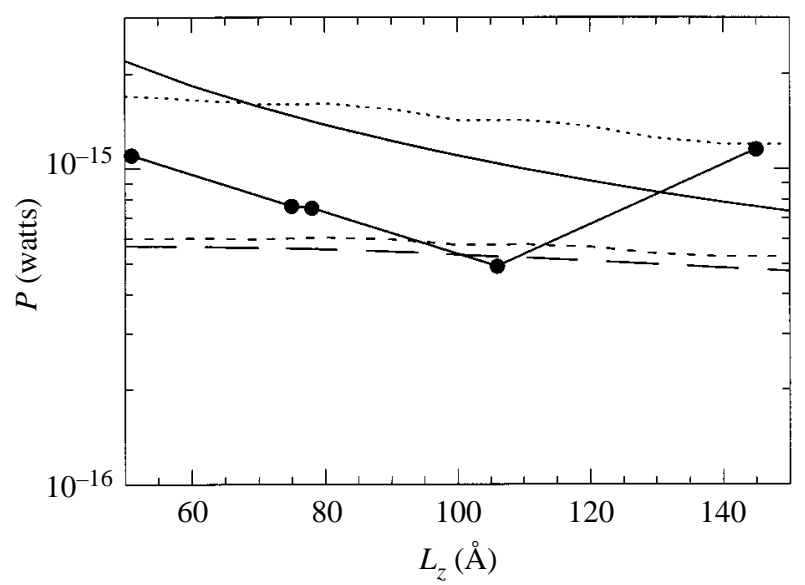

Fig. 4. The well-width dependence of the acoustic phonon power loss as obtained by using the approximate solution (solid curve), the exact result (dotted curve), static screening (short-dashed curve) and dynamic screening (long-dashed curve). The experimental data are shown by the solid line with circles marking the data points.

values for $\Xi$ and concluded that screening must be included. However, the theory would still not predict the well-width dependence of the experimental results.

Possible explanations for the discrepancies are that there is some electron population or scattering into another subband. However, the densities and temperatures at which the results are taken are too small for this to occur. It is possible that including another (empty) electron subband in the dielectric function would change the well-width dependence of the power loss theoretically, but this is unlikely because of the low frequency of the acoustic phonons.

To include the effect of many subbands, we may rewrite eqn (11) (ignoring any coupling) as

$$
P=\sum_{\boldsymbol{q}, i j} 2 \omega_{\boldsymbol{q}}\left[n T_{e}\left(\omega_{\boldsymbol{q}}\right)-n_{T}\left(\omega_{\boldsymbol{q}}\right)\right]\left|\boldsymbol{M}_{i j}^{\prime}(\boldsymbol{q}, 0)\right|^{2} \operatorname{Im}\left[-\chi_{i j}\left(\boldsymbol{q}_{\|}, \omega_{\boldsymbol{q}}\right)\right]
$$

with $i$ and $j$ as the initial and final subband indices, $\chi_{i j}\left(\boldsymbol{q}_{\|}, \omega_{\boldsymbol{q}}\right)$ similar to eqn (10) but including the subband energy difference [17] and

$$
M_{i j}^{\prime}(\boldsymbol{q}, \omega)=\sum_{m n} \varepsilon_{i j m n}^{-1}(\boldsymbol{q}, \omega) M_{m n}(\boldsymbol{q}),
$$

with $\left|M_{m n}(\boldsymbol{q})\right|^{2}$ similar to eqn (8) but including the correct transition and with the different phonon interactions treated separately. The dielectric function is now given by the matrix $\varepsilon_{i j m n}\left(\boldsymbol{q}_{\|}, \omega\right)=\delta_{i n} \delta_{j m}-$ $V_{i j m n}\left(\boldsymbol{q}_{\|}\right) \chi_{m n}\left(\boldsymbol{q}_{\|}, \omega\right)$. If $\varepsilon_{i j m n}\left(\boldsymbol{q}_{\|}, \omega\right)=\delta_{i n} \delta_{j m}$, i.e. $M_{i j}^{\prime}(\boldsymbol{q}, \omega)=M_{i j}(\boldsymbol{q})$, we again obtain the unscreened result.

Figure 5 compares the power loss for A, the unscreened and B, the screened cases for different electron densities. It can be seen that, although the power loss does indeed increase rapidly when a new subband is filled, this increase occurs at larger well widths for the electron densities in the experiments. Increasing the density does reduce the well width for which the increase occurs but the power loss per electron is also reduced. Including the effect of screening does reduce the power loss with only a marginal effect on the shape of the curve.

The theory predicts that there should be almost no well-width dependence of the power loss, whether screening is included or not, for the range of densities and well widths used. In most cases it would appear as though it is possible to use the unscreened power loss for comparison with experimental data as long as one of the low values of $\Xi$ is used, such as $7 \mathrm{eV}$. 

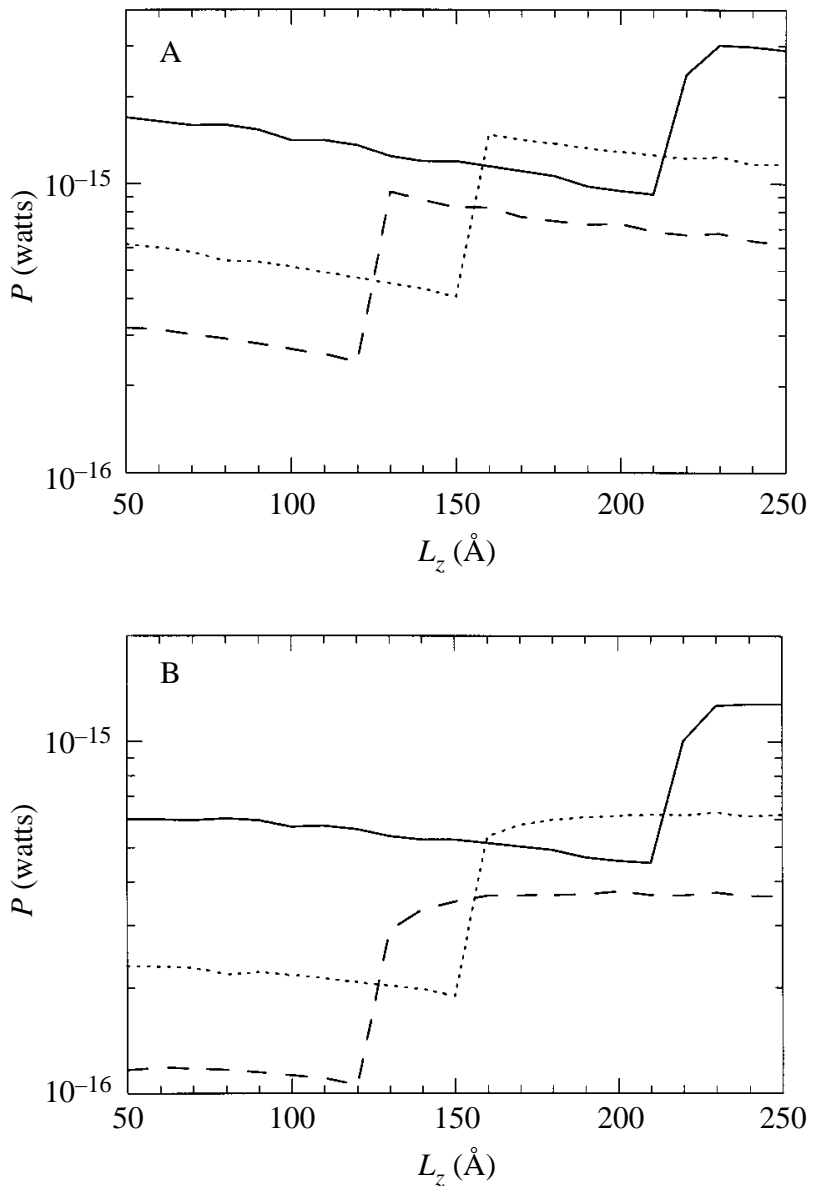

Fig. 5. A, The unscreened and $\mathrm{B}$, the screened acoustic phonon power loss against well-width for electron densities of $10^{12} \mathrm{~cm}^{-2}$ (solid curve), $2 \times 10^{12} \mathrm{~cm}^{-2}$ (dotted curve) and $3 \times 10^{12} \mathrm{~cm}^{-2}$ (dashed curve).

\section{Summary and conclusions}

Experimental studies of energy-loss rates of warm electrons via scattering from acoustic phonons in quantum-well structures have been presented. The results are compared with the predictions of the approximate calculations of both the unscreened 3D and the 2D analytical models. It is shown that the 2D model explains the well-width dependence for narrow wells, but overestimate the magnitude of the loss rates. The exact numerical calculation of the 2D model and the inclusion of static or dynamic screening fail to predict the observed power loss. One reason for this might be the infinite quantum-well approximation used in the theoretical models. In the extreme quantum limit, the confined electron-bulk acoustic phonon interaction is enhanced compared with the case in a real quantum well with finite barriers. As a result, a larger power loss than that observed experimentally is not totally unexpected. Also, all the existing theories use the bulk phonon approximation, which is obviously valid for wide wells. However, recent work on the acoustic phonon confinement in GaAs slabs in vacuum suggests that the electron-confined mode scattering rates for narrow wells may indeed be different from that obtained by using the bulk phonon approximation [18]. By how much the confinement of the acoustic phonons in a $\mathrm{GaAs} / \mathrm{Ga}_{1-x} \mathrm{Al}_{x}$ As system would change the scattering 
rates is an interesting problem that needs to be considered. Furthermore, the value for the sound velocity for GaAs for propagation in the $\langle 110\rangle$ direction that is used in the calculations has to be modified with the appropriate weighting to take account of the GaAlAs mode propagation. To our knowledge no theoretical study on the electron-acoustic phonon scattering exists in the literature which takes account of both the electron confinement in a finite well, and the confinement and the folding of the LA and the TA modes.

Acknowledgements-We are grateful to TUBITAK (TBAG-1193), and the British Council through its academic links scheme for financial support.

\section{References}

[1] H. Sakaki, K. Hirakawa, J. Yoshino, S. P. Svenson, Y. Sekiguchi, T. Hotta, and S. Nishi, Surface Sci. 142 , 306 (1984).

[2] K. Hirakawa and H. Sakaki, Appl. Phys. Lett. 49, 889 (1986).

[3] K. P. Martin, R. J. Higgins, and J. S. L. Rascal, Surface Sci. 196, 323 (1988).

[4] A. M. Kresschuk, M. Martinov Yu, T. A. Polyanskaya, I. G. Savd'ev, I. I. Saidashev, A. Shik Ya, and V. Yu Shmartsev, Solid State Commun. 65, 1189 (1988).

[5] Y. Kodaira, H. Kuwano, and K. Tsubaki, Appl. Phys. Lett. 54, 2474 (1988).

[6] D. R. Leadley, R. J. Nicholas, J. J. Harris, and C. T. Foxon, Solid State Electron. 32, 1473 (1989).

[7] Ç. Arikan, A. Straw, and N. Balkan, J. Appl. Phys. 74, 6261 (1993).

[8] N. Balkan, H. Çelik, A. J. Vickers, and M. Cankurtaran, Phys. Rev. B52, 17210 (1995).

[9] B. K. Ridley, Rep. Prog. Phys. 54, 169 (1991).

[10] R. Fletcher, J. J. Harris, C. T. Foxon, and R. Stoner, Phys. Rev. B45, 6659 (1992).

[11] M. E. Daniels, B. K. Ridley, and E. Emeny, Solid State Electron. 32, 1207 (1989).

[12] B. K. Ridley, Quantum Processes in Semiconductors (Oxford Science, Oxford, 1988).

[13] P. J. Price, J. Appl. Phys. 53, 6863 (1982).

[14] S. J. Manion and K. Hess, J. Appl. Phys. 62, 4942 (1987); S. J. Manion, M. Artaki, M. A. Emanuel, J. J. Coleman, and K. Hess, Phys. Rev. B35, 9203 (1987); S. Das Sarma, J. K. Jain, and R. Jalabert, Phys. Rev. B41, 3561 (1990).

[15] T. Kawamura, S. Das Sarma, R. Jalabert, and J. K. Jain, Phys. Rev. B42, 5407 (1990).

[16] A. J. Vickers, Phys. Rev. B46, 13315 (1992).

[17] Y. Ma, R. Fletcher, E. Zaremba, M. D’Iorio, C. T. Foxon, and J. J. Harris, Phys. Rev. B43, 9033 (1991).

[18] Se Gi Yu, K. W. Kim, M. A. Strascio, G. J. Iafrate, and A. Ballato, Phys. Rev. B50, 1733 (1994). 